\title{
Accelerating Taylor Bubbles within Circular Capillary Channels: Break-up Mechanisms and Regimes
}

\author{
Manolia Andredaki ${ }^{1}$, Anastasios Georgoulas ${ }^{1}$, Nicolas Miché ${ }^{1}$, Marco Marengo ${ }^{1}$ \\ ${ }^{1}$ Advanced Engineering Centre, School of Computing Engineering and Mathematics, Cockcroft Building, Lewes \\ Road, University of Brighton, Brighton BN2 4GJ
}

\begin{abstract}
In the present paper, an enhanced Volume of Fluid model is applied for the conduction of parametric numerical simulations, to investigate break-up phenomena of accelerating, elongated, vapour bubbles, within circular mini-channels. The effect of fundamental controlling parameters in the resulting break-up characteristics is investigated. Four different series of parametric numerical simulations of isolated vapour bubbles within mini-channels are performed, examining the effects of the imposed pressure difference between the inlet and the outlet of the channel, the surface tension, the effect of the applied heat flux as well as the initial liquid film thickness between the bubbles and the channel, on the developed vapour/liquid interface dynamics. The overall dimensionless number ranges examined are $6.76<\mathrm{We}<1474.7,0.007<\mathrm{Ca}<0.14,694<R e<12541$ and by introducing a modified Froude number in order to account for the flow acceleration, $1<F r^{*}<21.86$. These dimensionless number ranges are selected in order to overlap with experimental observations in zero-gravity Pulsating Heat Pipe experiments that constitute the motivation for the present numerical investigation. The proposed simulation results identify three prevailing regimes. A "full break-up" regime, a "partial break-up" regime and a "no break-up" regime. The entrainment of liquid droplets at the trailing edge of the vapour slugs is in most cases responsible for their subsequent "full break-up", into a leading and a trailing bubble, as it is identified from the numerical simulations. Moreover, the applied heat flux does not influence the resulting break-up regimes. Finally, these identified break-up regimes, are grouped together into a well-defined flow map with respect to the $W e$ and $F r^{*}$ numbers.
\end{abstract}

Keywords: Taylor Bubble flow, Accelerating flow, Mini-channels, Break-up mechanisms, VOF

\section{Introduction}

Taylor Bubble (TB) flow, also known as slug flow or slug-plug flow, is of fundamental importance in a variety of engineering and other industrial applications. Examples include but are not limited to chemical micro-process engineering, microfluidics, catalytic and multiphase reactors, blood flow in capillaries, on-chip cooling in supercomputers, miniaturized heat exchangers, fuel cells and bubble pumps [1]. This flow pattern consists of bulletshaped bubbles (vapour/gas slugs) separated by liquid plugs. A thin liquid film also separates these vapour/gas bubbles from the channel walls while connecting the successive liquid plugs. The proposed flow pattern, in most cases, constitutes a quasi-periodic, two-phase flow that occurs over a wide range of spatial scales ( $\mu \mathrm{m}$ - to $\mathrm{cm}$ - scale diameter channels), occupying a significant percentage of existing two-phase flow regime maps [2]. A wide number of experimental investigations has been focused in the flow visualisation of the TB regime (e.g. [3]-[8]). According to these studies, the main parameters that affect TB flows within capillaries are the flow velocity, the pressure drop in the liquid plug and around the bubble as well as the thickness of the surrounding liquid film.

TB flows in capillary channels have also been investigated numerically applying a variety of different modelling treatments. In relatively early studies (e.g. [9]) the Taylor bubble is considered to be of an idealised shape, consisting of two half-spheres at the ends connected to a cylindrical middle part. Then, the liquid flow around the bubble is simulated, assigning a free slip boundary condition at the surface that represents the interface between the liquid and gaseous phases. In another early numerical investigation, Taha and Cui [10] applied the VOF method to study the flow of TBs in circular capillary tubes, using a geometric reconstruction technique to track the interface between the bubble and the liquid. In a later work by Gupta et al. [11], a similar model is applied in conjunction with some special meshing treatments, in order to reduce spurious velocities and to adequately capture the liquid film dynamics. Two different CFD methodologies, based on the VOF method, are also used in the work of Asadolahi et al. [12], to enable Taylor bubbles with controlled bubble and slug lengths to be simulated numerically. In the first approach, the bubbles and slugs of desired length were created by employing a transient inlet boundary condition, which allowed a known amount of gas and liquid to enter the computational domain alternately. By having a sufficiently long solution domain, fully 
developed values can be obtained and a quasi-steady state for the two-phase flow can be reached. In the second approach a moving domain is used to model the periodic TB flow in a unit cell. The hydrodynamic and heat transfer results obtained from the two approaches were found to be very similar to each other. In another work by LizarragaGarcia et al. [13], a prediction of TB velocity for inclined pipes with stagnant liquid for a wide range of properties and inclination angles was developed, utilising a wide number of parametric 3D CFD Direct Numerical Simulations with Level Set as the Interface Tracking Method. Shaban and Tavoularis [14] performed simulations of TBs with turbulent wakes using a combination of the VOF method and the Detached Eddy Simulation (DES) approach. They found that the recirculating vortex in the rear wake of a leading bubble accelerates the trailing bubble coalescence.

Only in the high Re cases of [3], [7], [14] some intense interface instabilities and break-ups are reported. However, even in these cases, the focus of the proposed works was not on the investigation of such interfacial phenomena and these are not discussed or analysed. The main characteristics from all the considered previous investigations, are summarised in Table 1.

Table 1. Main characteristics from previous investigations on TBs.



In the majority of the aforementioned applications that are summarised in Table 1, TBs are usually moving with a steady velocity towards a particular direction. However, there are cases where TB flows might be oscillating in nature, with flow reversals leading in transient, accelerating and/or decelerating stages. The nature of these oscillations might be either pressure driven as in the case of adiabatic flows (e.g. [15]) or also related to local thermodynamic phenomena within two-phase, closed-loop, wickless heat pipes, such as ThermoSyphons (TS) and Pulsating Heat Pipes (PHPs), that constitute the motivation of the present numerical investigation [16]. Such twophase flow heat dissipation systems that are based on the local phase change of a confined working fluid, have originated from the demand for increasingly higher performances of electronic equipment. As an example of these systems, Mangini et al. [16] tested a novel concept of a hybrid TS/PHP with a diameter bigger than the capillary limit, both on ground and in hyper/micro gravity conditions, during the 61st ESA Parabolic Flight Campaign. The proposed device was made of an aluminium tube, with Internal Diameter (ID) of $3 \mathrm{~mm}$, bent into a planar serpentine and filled with FC-72. The proposed setup with some indicative flow visualization snapshots during the normal gravity, hyper-gravity and microgravity stages of a single parabola, are illustrated in Figure 1. During the $1 \mathrm{~g}$ and $1.8 \mathrm{~g}$ periods, the developed two-phase flow within the visualisation channel of the proposed device is stratified, while during the microgravity period $(0 \mathrm{~g})$ the flow is transformed into a slug-plug flow. However, during the microgravity period and particularly during the decelerating and accelerating stages before and after the occurrence of a 
flow reversal, the high-speed snapshots from the experiments reveal the development of intense capillary waves in the liquid film and other types of inertial perturbations at the interface between the liquid and vapour phases that in most cases lead to the break-up of the vapour slugs, in the vicinity of their trailing edges, either into two subsequent elongated vapour slugs (denoted with A in Figure 1) or into a main elongated vapour slug with small bubbles detaching from its trailing edge (denoted as B in Figure 1). In other cases, intense capillary waves are evident in the liquid film between the bubbles and the channel wall without any break-up event identified (denoted with $\mathrm{C}$ in Figure 1). These phenomena are observed really often in such experiments of accelerating/decelerating TB flows and for a variety of experimental conditions. For example, in the recent work by Pietrasanta et al. [17], flow pattern transitions and bubble break-up and coalescence events have been investigated experimentally, in a passive two phase wickless capillary loop, where the mass flow rate is intrinsically not controllable. Modified Froude, Weber and Bond numbers have been introduced, considering the actual acceleration of the fluid and the length of the bubble as merit parameters for the transitions. However, the nature and underpinned formation mechanisms of such interfacial instabilities are not yet fully identified and quantified.
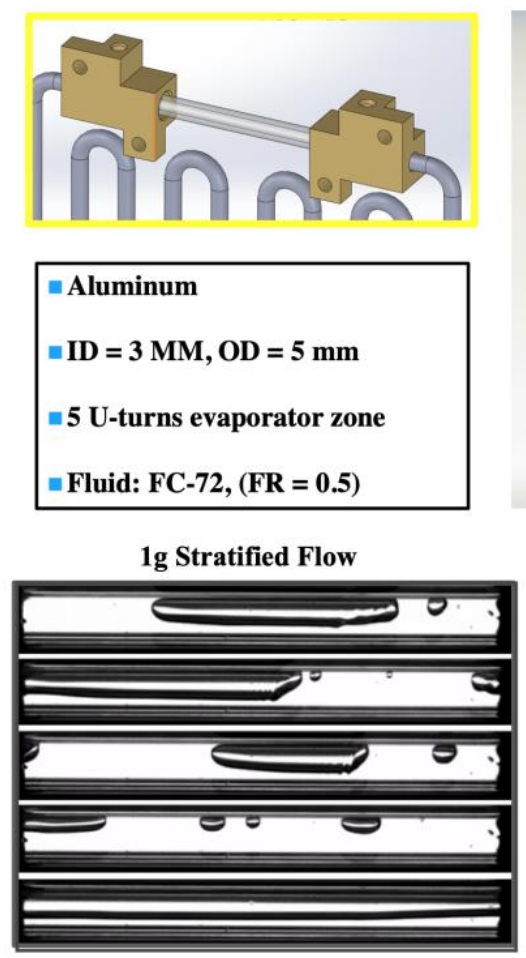



Figure 1. Experimental two-phase flow visualization within a hybrid TS/PHP [16], [19].

To better understand interfacial instabilities, bubble break-up and/or coalescence phenomena in accelerating TB flows within mini-channels, the fundamental problem of a single Taylor bubble should be thoroughly investigated for a variety of operating conditions, laying a solid foundation for the analysis of more complex cases, where a sequence of TBs is examined. The present investigation therefore, aims to give further insight on such dynamic vapour/liquid interfacial phenomena by identifying and quantifying break-up regimes in the case of isolated elongated vapour slugs within circular mini-channels in adiabatic as well as saturated flow boiling conditions within a mini-channel, starting with the same characteristics as the mini-channel branches of the previously described hybrid TS/PHP device microgravity experiments [16] that constitute the motivation of the present numerical investigation. The ultimate aim is to construct a flow map of TB break-up regimes with respect to the global flow conditions. This can then lead to appropriate conditional relationships that can be incorporated in the future, as break-up sub-models, into advanced 1-D codes like the ones that are described in [18]. The overall dimensionless number ranges examined in the present numerical investigation are $6.76<W e<1474.7,0.007<C a<0.14,694<R e<12541$ and by introducing a modified Froude number in order to account for the flow acceleration, $1<F r^{*}<21.86$. These dimensionless number ranges have been selected in order to overlap with the experimental conditions in the zero-gravity Pulsating Heat pipe experiments reported and analysed in [16] and [19]. In more detail, the same channel diameter (3mm) and same working fluid (FC- 
72) as in [16] and [19] are selected, for the majority of the runs in the proposed parametric numerical investigation. The imposed pressure differences are selected in order to result in a range of maximum velocities that overlaps with the range of maximum flow velocities that were estimated from the experimental high-speed video snapshots in [16] and [19] $\left(0.1 \mathrm{~m} / \mathrm{s}<U_{\max }<1 \mathrm{~m} / \mathrm{s}\right)$. In order to further extend the initially selected ranges of the parametric numerical investigation, an additional fluid (Ethanol) that is widely used in such experimental campaigns, a smaller channel diameter $(2 \mathrm{~mm})$ as well as additional imposed pressure differences were tested. The overall details and conditions are summarised in Table A in the Appendix.

\section{Investigation Methodology}

\subsection{Numerical Simulation Framework}

An Enhanced VOF method that has been previously developed in the context of OpenFOAM CFD Toolbox [20] is applied in order to perform a series of parametric numerical experiments, aiming to understand the various break-up mechanisms and characteristics. Enhancements with respect to the original VOF method in OpenFOAM include a treatment for spurious velocities dampening in the vicinity of the interface (a well-known defect of VOF methods), implementation of a more accurate dynamic contact angle treatment, in order to account for wettability effects in case of a dry spot, implementation of the energy equation as well as a phase-change model to account for boiling and condensation phenomena and finally implementation of conjugate heat transfer between solid and liquid domains. It should be mentioned at this point that despite the fact that wettability is taken into consideration in the proposed solver, for the purposes of the present investigation that is focused on the TB regime, in all of the conducted simulations a liquid film is always present between the bubbles and the wall of the cylindrical domains (i.e. fully wetting conditions). Therefore, this aspect of the solver is not used for the present simulations. Furthermore, since the focus of the present study is on interface dynamics, only flow domains are considered even for the diabatic cases, without considering any conjugate heat transfer. The proposed numerical simulation framework, has been extensively validated in the past against existing analytical solutions and experimental data on adiabatic and diabatic bubble and droplet dynamics by the present Authors' research team ([21]-[24]). Some, of these validation results are represented in Figure 2, since they prove that all of the underpinned physics are accurately captured by the proposed improved VOF solver (i.e. interface dynamics, evaporation). As it can be observed, the proposed VOF-based numerical modelling framework accurately predicts adiabatic and diabatic bubble and droplet dynamics, in general, including phase-change due to boiling. Despite the fact that all these previous validation runs are not for Taylor bubble flows, they prove that interfacial phenomena, such as bubble pinch-off (break-up) from submerged orifices as well as evaporation due to boiling that are important for the present investigation, are accurately captured by the solver. More relevant validation cases to the present investigation are considered in Section 2.3. 




(a)



(c)



(b)


(d)

Figure 2. Comparison between (a) numerical simulation and experimental results for an air bubble growing quasi-statically from a submerged orifice into a stagnant liquid domain [21], (b) conjugate heat transfer numerical simulation results and experimental measurements on droplet impact cooling [23], (c) numerical simulation and analytical solution results for vapour bubbles growing in superheated liquid domains [22] and (d) numerical simulation results and experimental results for a bubble evaporating and detaching from a superheated plate in case of saturated pool boiling [22]. All the presented numerical simulations in this figure have been conducted in previous investigations of the present Authors' research team with the same solver as the one that is used in the present paper.

\subsection{Numerical Method}

\subsubsection{Governing Equations}

In this section, the governing equations for mass, momentum, energy, and volume fraction are presented. It should be mentioned that liquid and vapour phases are both treated as incompressible, Newtonian fluids. Since for all the numerical simulations that are presented in the present paper, only fluid domains are considered, the theoretical background does not include the conjugate heat transfer part of the proposed numerical simulation framework. Details for the conjugate heat transfer version of the numerical simulation framework can be found in [23] and [24]. Moreover, in all of the considered cases there is always a liquid film around, between the bubble and the wall of the channels (i.e. no vapour/liquid/solid wall triple line is present). Therefore, the details for the dynamic contact angle implementation of the proposed numerical simulation framework are not presented in the present paper. The reader is hence directed to [25], for further details on the dynamic contact angle implementation of the utilised numerical simulation framework.

The mass conservation equation is given as:

$$
\boldsymbol{\nabla} \cdot(\boldsymbol{\rho} \overrightarrow{\mathbf{U}})=\dot{\boldsymbol{\rho}}
$$

where $\vec{U}$ is the fluid velocity and $\rho$ is the bulk density. The source term on the right-hand side, $\dot{\rho}$, accounts for the phase change. The conservation of momentum is given by the following equation: 


$$
\frac{\partial}{\partial t}(\rho \vec{U})+\nabla \cdot(\rho \vec{U} \vec{U})-\nabla \cdot\left\{\mu\left[\nabla \vec{U}+(\nabla \vec{U})^{T}\right]\right\}=-\nabla p+\vec{f}_{S T}+\vec{f}_{g}
$$

where $p$ is the pressure and $\mu$ is the bulk dynamic viscosity. The momentum source terms on the right-hand side of the equation account for the effects of surface tension $\left(\vec{f}_{S T}\right)$ and gravity $\left(\vec{f}_{g}\right)$, respectively. The surface tension term is modelled according to the classical approach of Brackbill et al. [26]. The conservation of energy balance is given by the following equation:

$$
\frac{\partial}{\partial t}\left(\rho c_{p} T\right)+\nabla \cdot\left(\vec{U} \rho c_{p} T\right)-\nabla \cdot(\lambda \nabla T)=\dot{h}
$$

where $c_{p}$ is the bulk heat capacity, $T$ is the temperature field and $\lambda$ is the bulk thermal conductivity. The source term on the right-hand side of the equation $(\dot{h})$ represents the contribution of the enthalpy of evaporation/condensation or else the cooling/heating associated with the latent heat of the phase-change. The volume fraction $\alpha$ is advected by the flow field by the following equation:

$$
\frac{\partial \alpha}{\partial t}+\nabla \cdot(\alpha \vec{U})-\nabla \cdot\left(\alpha(1-\alpha) \vec{U}_{r}\right)=\frac{\dot{\rho}}{\rho} \alpha
$$

The source term on the right-hand side of Equation (4) is needed since, due to the local mass source terms, the velocity field is not free of divergence. Interface sharpening is very important in simulating two-phase flows of two immiscible fluids. In OpenFOAM the sharpening of the interface is achieved artificially by introducing the extra compression term $\left.\nabla \cdot\left(\alpha(1-\alpha) \vec{U}_{r}\right)\right)$ in Equation (4). $\vec{U}_{r}$ is an artificial compression velocity:

$$
\vec{U}_{r}=\vec{n}_{f} \min \left[C_{\gamma} \frac{\phi}{s_{f}}, \max \left(\frac{\phi}{s_{f}}\right)\right]
$$

The divergence of the compression velocity $\vec{U}_{r}$, ensures the conservation of the volume fraction $\alpha$, while the term $\alpha(1-\alpha)$ limits this artificial compression approach only in the vicinity of the interface, where $0<\alpha<1$ [27]. The level of compression depends on the value of $C_{\gamma}$ ([27], [28]). For the simulations of the present investigation, initial, trial simulations indicated that a value of $C_{\gamma}=1$ should be used, in order to maintain a quite sharp interface without at the same time having unphysical results. Finally, $\vec{n}_{f}$ is the normal vector of the cell surface, $s_{f}$ is the cell surface area and $\phi$ is the mass flux.

Finally, the bulk fluid properties $\gamma_{b}$ are computed as the averages over the liquid $\gamma_{l}$ and vapour $\gamma_{v}$ phases, weighted with the volume fraction $\alpha$ :

$$
\gamma_{b}=\alpha \gamma_{l}+(1-\alpha) \gamma_{v}
$$

\subsubsection{Phase Change Model}

The applied phase change model that was implemented in the improved OpenFOAM VOF solver of the present investigation will be described briefly in this sub-section. In the case of evaporation, the evaporating mass flux at the liquid-vapour interface $\mathrm{j}_{\mathrm{evap}}$ is calculated from the following equation:

$$
\mathbf{j}_{\text {evap }}=\frac{T_{\text {int }}-T_{\text {sat }}}{\mathbf{R}_{\text {int }} \mathbf{h}_{\mathrm{lv}}}
$$

where $T_{\text {int }}$ is the temperature of the interface, $T_{\text {sat }}$ is the saturation temperature, $R_{\text {int }}$ is the interfacial heat resistance and $h_{l v}$ is the latent heat of evaporation at the saturation temperature. The amount of liquid that evaporates is calculated 
locally and the resulting source term field is smeared over a few cells, in order to avoid numerical instabilities. The evaporating mass is taken away on the liquid side of the interface and reappears on the vapour side, following the process suggested in [29]. Further details on the proposed phase-change model implementation and its validation against analytical solutions and literature available experimental data can be found in [22].

\subsubsection{Simulation Parameters}

All the numerical simulations of the present work were performed with the finite volume-based CFD code OpenFOAM (version 2.2.1), using a user-enhanced version of its original VOF-based solver "interFoam". For pressurevelocity coupling, the PISO (Pressure-Implicit with Splitting of Operators) scheme is applied. The transient terms in the equations are discretised using a second order, bounded, implicit scheme (Euler). The calculation time step is controlled by setting the maximum Courant number to 0.2. With this adaptive time-stepping technique, the time step is automatically varied from approximately $10^{-9}$ to $10^{-6} \mathrm{~s}$ for the overall simulation cases that are presented in the present paper. The gradient terms are discretised using a second-order, Gaussian integration with linear interpolation (Gauss linear). For the divergence terms, different discretisation schemes are applied for each term in the equations. In more detail, the convection term of Equation (2) is discretised using a "Gauss upwind" scheme. The $\nabla \cdot(\alpha \vec{U})$ term of Equation (4) is discretised using the "Gauss vanLeer" scheme, while the $\nabla \cdot\left(\alpha(1-\alpha) \vec{U}_{r}\right)$ term is discretised using the "Gauss interfaceCompression" scheme which ensures the boundedness of the calculated volume fraction field. Finally, all Laplacian terms are discretised using the "Gauss Linear Corrected" scheme. The divergence term of Equation (3) is discretised using a "Gauss linear" scheme. Further details on the proposed discretisation schemes can be found in OpenFOAM documentation [20]. It should be mentioned that this was the optimum combination of discretisation schemes in order to maintain a balance between accuracy, convergence and numerical stability during the computations. Due to the large number of simulations the High-Performance Computing facility of the School of Computing Engineering and Mathematics, in the University of Brighton was used for the computations. Indicatively, for each case considering both the initial single-phase flow stage as well as the subsequent two-phase flow stage of the simulation, a total duration of 5 days of calculations were needed, occupying from 8 up to 16 cores for each run.

\subsubsection{Dimensionless Numbers}

The overall dimensionless numbers that are used in the present investigation are given by the following formulae:

$$
\begin{aligned}
\mathrm{We} & =\frac{\rho U^{2} \mathrm{D}}{\sigma} \\
\mathrm{Ca} & =\frac{\mu U}{\sigma} \\
\mathrm{Re} & =\frac{\rho \mathrm{DU}}{\mu} \\
F r^{*} & =\frac{\rho U^{2}}{\Delta P}
\end{aligned}
$$

where $\rho$ is the liquid density, $U$ is the maximum flow velocity, $D$ is the channel diameter, $\sigma$ is the surface tension, $\mu$ is the liquid dynamic viscosity and $\Delta P$ is the imposed pressure difference between the inlet and the outlet of the channel. The proposed modified Froude number in equation (11), incorporates the effects of the imposed pressure difference between the inlet and the outlet of the channel and therefore of the global acceleration of the confined two-phase flow in the longitudinal direction. The derivation of the proposed equation (Equation 11), starting from the classical Fr number relationship, can be found in the Appendix.

\subsection{Numerical Method Validation}

Since the focus of the present investigation is on TB break-up phenomena, it was deemed necessary to conduct an additional validation against literature available experiments on TB development in a mini-channel T-junction [30], despite the fact that the applied, enhanced VOF-based numerical model has been extensively validated previously, 
against existing analytical solutions and experimental data on adiabatic and diabatic bubble dynamics (mainly for bubbles growing quasi-statically from orifices into stagnant liquid domains and boiling bubbles growing and detaching from superheated plates into saturated stagnant liquid pools), by the Authors' research team. This additional validation in the present sub-section of the paper demonstrates that the proposed VOF-based numerical solver can also accurately predict such, more dynamic, two-phase flow and interface instability phenomena. The initial conditions and the characteristics of the selected experimental runs that were reproduced numerically are summarised in Table 2. The fluids used are water and air at ambient conditions. Full wetting conditions are applied in the channel walls. As it can be observed from Table 2, for all three cases the T-junction channel diameter is $D_{h}=1 \mathrm{~mm}$. At this point, it should be mentioned that these experiments were selected as being quite close to the dimensionless parameter range of the targeted parametric numerical investigation. There is not an overlap in the dimensionless parameters, however, all of them are either really close to the lower limit of the dimensionless parameter ranges of the present parametric investigation, or at least of the same order of magnitude. The corresponding dimensionless parameter values for these three validation runs are summarised in Table 3 .

The 3D computational domain, the computational mesh and the applied boundary conditions for the proposed validation cases are illustrated in Figure 3. It should be mentioned that the utilised mesh density resulted from a mesh independence study.



Figure 3. Computational domain, mesh and boundary conditions, utilised in the validation runs.

Table 2. Initial conditions of validation experiments reported in [30].

\begin{tabular}{|l|l|l|l|}
\hline & $\mathbf{U}_{\text {water }}[\mathbf{m} / \mathbf{s}]$ & $\mathbf{U}_{\text {air }}[\mathbf{m} / \mathbf{s}]$ & $\mathbf{D}_{\mathbf{h}}[\mathbf{m}]$ \\
\hline Case 1 & 0.106 & 0.344 & 0.001 \\
\hline Case 2 & 0.531 & 0.068 & 0.001 \\
\hline Case 3 & 0.318 & 0.242 & 0.001 \\
\hline
\end{tabular}


Table 3. Dimensionless numbers values for the validation experiments of Table 2 [30].

\begin{tabular}{|c|c|c|c|c|}
\hline & Fr* & We & Ca & Re \\
\hline Case 1 & 0.06 & 0.156 & 0.001 & 106 \\
\hline Case 2 & 0.94 & 3.916 & 0.007 & 531 \\
\hline Case 3 & 0.84 & 1.405 & 0.004 & 318 \\
\hline
\end{tabular}

A macroscopic comparison of the numerical model predictions with the corresponding experimental data for the spatial and temporal evolution of the interface between the liquid and gas phases is conducted in Figure 4. A length legend has been added in each case for a more quantitative representation of the resulted bubble size as well as of the resulted distance between subsequent bubbles.


Figure 4. Comparison of experimental (left) [30] and numerical (right) results (present investigation), for the three different cases of TB flow generation in the considered mini-channel T-junction.

As it can be observed from Figure 4, the numerical model accurately captures the development of the different twophase flow regimes in relation to the applied gas/liquid flow rates, at the inlets. The numerically predicted bubble generation frequency as well as the generated TB dimensions in each case, show very close agreement with the corresponding experimental data. In order to have an even more quantitative comparison, the bubble generation frequencies that resulted from the numerical simulations are directly compared with the ones from the experiments in Table 4. 
Table 4. Comparison between numerically predicted (present investigation) and experimentally measured [30], bubble generation frequencies.

\begin{tabular}{|c|c|c|c|}
\hline & Numerical $\boldsymbol{F}_{\text {bubble }}[\mathbf{H z}]$ & Experimental $\mathbf{F}_{\text {bubble }}[\mathbf{H z}]$ & Difference [\%] \\
\hline Case 1 & 91 & 88.5 & 3 \\
\hline Case 2 & 222 & 204 & 9 \\
\hline Case 3 & 189 & 200 & 6 \\
\hline
\end{tabular}

Hence, considering also the validation cases presented in previous investigations by the Authors' research team [21]-[23], it can be concluded that the proposed numerical modelling framework can be safely applied for the purposes of the present investigation.

\section{Simulations of Bubble Break-up and Regimes}

In the present section, the adopted numerical simulation framework is further applied for the conduction of a wide series of parametric numerical simulations. The main aim is to identify and quantify the effects of fundamental flow controlling parameters in the resulting interface dynamics, for the case of isolated elongated vapour slugs within adiabatic as well as heated mini-channels. As mentioned previously, two different working fluids (FC-72 and Ethanol) and two different channel diameters $(3 \mathrm{~mm}$ and $2 \mathrm{~mm}$ ) are considered. The overall parameters that are varied are: i) the Imposed Pressure Difference (IPD) between the inlet and the outlet of the considered minichannels (i.e. the driving force of the generated flows), ii) the Surface Tension (ST), iii) the Applied Heat Flux (AHF) in the central part of the considered circular mini-channels and iv) the Initial Liquid Film Thickness (ILFT) between the vapour slugs and the channel wall.

For the cases with channel diameter of $3 \mathrm{~mm}$ and FC-72 as the working fluid, IPD values ranging from $15 \mathrm{~Pa}$ up to $90 \mathrm{~Pa}$ were used. However, for the cases with $2 \mathrm{~mm}$ diameter and FC-72 as the working fluid the IPD values ranged from $15 \mathrm{~Pa}$ up to $180 \mathrm{~Pa}$. Finally, for both of the examined channel diameters, in the cases with Ethanol as the working fluid, IPD values from $60 \mathrm{~Pa}$ up to $400 \mathrm{~Pa}$ were used. At this point, it should be mentioned once more that the initially applied IPD value ranges were selected in order to result to typical flow velocities that were estimated in the PHP experiments of [16] and [19] and they were further extended to cover an even wider parameter framework.

As for the variation of the ST, this was conducted in a "virtual fluid" manner, since starting from real fluid properties for FC72 and Ethanol as base cases, only the ST values were varied keeping the rest of the liquid and vapour phase properties unchanged. Therefore, "virtual fluids", not real fluids, are considered for this purpose.

All the cases, described above, are considered to be adiabatic with the fluid properties for the liquid and vapour phases taken at a saturation state. For the last series of parametric numerical simulations, 12 additional cases are simulated, selecting 6 different runs from the previously described simulations and applying two different heat flux values in the central part of the considered circular mini-channel. This is done in order to explore also the resulting interface dynamics for the case of diabatic flows where the considered TBs are also subject to elongation due to liquid film evaporation, while they are moving towards the downstream end of the channel.

Finally, the effect of the ILFT was also examined since it needs to be applied as an assumed initial condition, estimated from the observed liquid film thickness values in the zero-gravity Pulsating Heat pipe experiments reported and analysed in [16] and [19].

The overall parametric analysis that was conducted for the purposes of the present investigation consists of a total number of 89 simulations. Initially, a prescribed pressure difference is imposed between the inlet and the outlet and a single-phase simulation is conducted in order for the hydrodynamic and, where appropriate, the initial thermal boundary layers to reach a quasi-steady state within the channels. Then, a vapour slug is patched close to the inlet of the channel that is carried away downstream by the previously developed liquid crossflow. The initial vapour slug length in all cases is taken as three times the considered channel diameter, for consistency. At this point, it should be mentioned that the initial bubble length might also be a controlling parameter in the investigated phenomenon. However, this falls outside the scope of the present parametric numerical investigation and it will be address separately in a future investigation. The ILFT for the base case is taken as $50 \mu \mathrm{m}$. In all cases the bubbles are initiated at a distance of $1.55 \mathrm{~mm}$ from the inlet of the channel, as a combination of a central cylindrical core with two superimposed spheres at the two ends. As mentioned previously, that the geometric (i.e. $3 \mathrm{~mm}$ channel diameter of the base case) as well as the range of the operating (i.e. IPD) and initial conditions (i.e. working fluids 
for the base cases, ILFT values) have been selected in order to be as close as possible to the experimental measurements of Mangini et. al. [16], [19]. The main characteristics for the overall runs are summarised in the Table A in the Appendix.

Finally, it should be highlighted that a constant pressure difference is imposed between the considered channel inlets and outlets as the driving force of the flow within the channels instead of a constant flow rate, in order to focus on accelerating TBs where inertial perturbations are present and might lead to Taylor Bubble break-up events, as observed in the accelerating and/or decelerating two-phase flow stages in the experiments of Mangini et al. [16], [19].

\subsection{Computational Set-up}

Since the focus of the investigation is on elongated vapour slugs from a typical TB flow regime in the absence of gravitational acceleration, a 2D axisymmetric computational domain was constructed for the proposed parametric analysis. As it can also be seen from Figure 1, in the absence of gravitational acceleration, the flow switches to a slug plug regime that is mainly axisymmetric. Some 3D features and deviations from a perfect symmetry are indeed present however since the main break-up regimes is the focus of the present investigation, this initial assumption is justified. Furthermore, it would practically impossible due to the prohibitively increased computational cost to perform the present parametric numerical investigation performing 3D runs. The utilised computational domain, mesh and boundary conditions are illustrated in Figure 5, for the $3 \mathrm{~mm}$ diameter channel. As it can be seen, a wedge type geometry was constructed representing a $5^{\circ}$ section of the corresponding $3 \mathrm{D}$ domain in the considered physical problem. As mentioned previously, two different circular channels are considered with diameters $3 \mathrm{~mm}$ and $2 \mathrm{~mm}$, respectively, having a total length of $90 \mathrm{~mm}$. A non-uniform, structured computational mesh with local cell clustering close to the wall of the channel was adopted in each case, consisting of 840,000 (3 mm diameter channel) and 666,000 (2 mm diameter channel) hexahedral cells, respectively. A minimum cell size of $1 \mu \mathrm{m}$ in the vicinity of the channel wall and a maximum cell size of $15 \mu \mathrm{m}$ close to the axis of symmetry were selected after a detailed mesh independence study that is presented in the next sub-section (Sub-section 3.2). The length of $90 \mathrm{~mm}$ was selected, in order to safely allow the initiated vapour slugs to travel downstream from their initiation point for a certain distance and hence to capture the resulted liquid film dynamics and the associated interface instabilities, but mainly the potential break-ups of the bubbles due to the inertial effects from the imposed pressure drops (i.e. longitudinal accelerations). At the solid walls, a no-slip velocity boundary condition was used with a fixed flux pressure boundary condition for the pressure values. As also mentioned previously fully wetted conditions are applied for the volume fraction values as a thin liquid film always exists between the vapour bubbles and the channel wall. For the diabatic cases, a fixed uniform temperature gradient that corresponds to the required heat flux value was applied at the heated part of the channel wall, while a zero temperature gradient was applied in the non-heated part. A pressure inlet boundary condition was used for the inlet and a pressure outlet boundary for the outlet. For all cases the relative outlet pressure was kept to zero and the relative inlet pressure was varied accordingly in each case, in order to impose the required pressure difference between the inlet and outlet of the channel. For the adiabatic runs, the fluid properties are selected at a saturation equilibrium point. For the diabatic runs, saturated flow boiling conditions are assumed. Therefore, the fluid properties are also taken at the corresponding saturation equilibrium point and the temperature of the liquid that enters from the inlet boundary is taken to be equal to the saturation temperature. 




Figure 5. Computational domain, mesh and boundary conditions, for the $3 \mathrm{~mm}$ diameter channel.

\subsection{Mesh Independence Study}

In order to adopt the utilised computational mesh characteristics for the simulations presented in the current investigation, a mesh-independence study was carried out. In more detail, three different mesh densities were used for each channel diameter that indicatively (for the case of $3 \mathrm{~mm}$ diameter channel) consisted of of 210000, 840000, and 1890000 cells. The adopted mesh consists of 840000 cells that constituted the considered mesh independent solution, for the purposes of the present parametric numerical investigation. Since the simulated TBs involve transient phenomena, such as droplet encapsulation into vapour bubbles, partial and full bubble break-up events as well as other kinds of interface instabilities, the mesh independency checks were conducted focusing on such events. Indicatively, a partial break-up event that involved small satellite bubbles that are generated at the trailing edge of the TBs is compared between the adopted (medium) mesh and the coarser and finer meshes, in Figure 6. 




Figure 6. Comparison between the numerical model predictions using the adopted mesh (middle) vs the coarse mesh (top), the fine mesh (bottom) for the same three subsequent time instances, after the initiation of the elongated vapour bubble.

As it can be observed, the adopted mesh characteristics ensure a mesh independent solution that captures the satellite bubble formation due to a partial break-up event as well as the formation of capillary waves in the liquid film towards the end of the TBs. Therefore, the adopted mesh can be safely used for the purposes of the present parametric numerical investigation.

\section{Parametric Numerical Simulations}

The effect of each of the examined controlling parameters is illustrated indicatively in the present section, comparing the identified break-up regimes for some of the overall runs that were conducted for the purposes of the present parametric numerical investigation. At the end of the present section a dimensional analysis is conducted considering all of the 89 cases, which leads to the composition of a dimensionless flow map, where the resulted breakup regimes are grouped according to the global flow conditions. The overall cases simulated, their main flow characteristics as well as the considered dimensionless parameter values are presented in Table A in the Appendix. The overall dimensionless number ranges examined in the present numerical investigation have been summarised in Section 1.

\subsection{Effect of Imposed Pressure Difference (IPD)}

The first parameter that is varied is the IPD between the inlet and the outlet of the considered mini-channels, as this constitutes the main driving force of the examined, accelerating two-phase flows. In Figure 7, this effect is illustrated qualitatively utilising three cases with a different IPD at the ends of the $3 \mathrm{~mm}$ diameter mini-channel. In more detail, Figures 7 (a), (b) and (c) depict the spatial and temporal evolution of the considered TB when the IPD is $15 \mathrm{~Pa}, 45 \mathrm{~Pa}$ and $70 \mathrm{~Pa}$, respectively. Four successive time instances of the accelerating TB within the mini-channel are shown in each case. Velocity magnitude contours are also superimposed in a central, longitudinal section within the channel. The black coloured line marks the liquid/vapour interface on the same central, longitudinal section along the 
channel. The 3D interface is shown with an opacity of just 35\%, in order to also visualise the velocity field within the bubble, at the proposed longitudinal section. The distance from the inlet of the considered channel is also indicated in each case, in order to highlight the relative position of the bubble within the channel in each time instance. This also provides a measure of the bubble dimensions in each case. Finally, the corresponding dimensionless parameters for each of the presented cases are given in detail in the figure caption.

For all three presented IPD cases, the rest of the examined fundamental controlling parameters in the present parametric analysis, i.e. the utilised working fluid, the ILFT, the ST and the AHF are kept constant $\left(D_{\text {channel }}=3\right.$ $\left.\mathrm{mm}, \mathrm{FC}-72, \Delta_{\text {film }}=50 \mu \mathrm{m}, \sigma=0.0084 \mathrm{~N} / \mathrm{m}, q^{\prime \prime}=0.0 \mathrm{~W} / \mathrm{m}^{2}\right)$. The overall IPD values examined for the purposes of the present parametric investigation range from $15 \mathrm{~Pa}$ up to $180 \mathrm{~Pa}$ with an average step of $10 \mathrm{~Pa}$, since in some cases steps as small as $5 \mathrm{~Pa}$ were used. Different values of IPD were tested for both of the considered channel diameters as well as for both of the considered working fluids (please see Table A in the Appendix for more details).

As it is obvious from Figure 7, even small variations in the IPD can lead to significant differences in the resulting bubble dynamics. In the first case, where the IPD value is $\Delta P=15 \mathrm{~Pa}$ (Figure 7 (a)), quite intense capillary waves are evident within the liquid film that surrounds the elongated vapour slug, especially close to its trailing edge. These capillary waves are evident up to the end of the simulated flow period from the initialisation (patching) of the vapour bubble close to the inlet of the channel, without any kind of bubble break-up being observed. At this point, it should be mentioned that for all cases considered the total duration of the simulated two-phase flows was selected to be $90 \mathrm{~ms}$. This observed behaviour of the simulated TB is referred to as a "No Break-up (NB)" regime in the rest of the present paper and it constitutes one of the three in total observed regimes. This regime corresponds to observation $\mathrm{C}$ in the experimental snapshots of Figure 1. Finally, it is important to highlight here that the VOFbased numerical solver that has been used in the present investigation has been treated accordingly by the Authors' research team, in order to filter out spurious interfacial velocities [31]. Therefore, the resulted capillary waves do not correspond to numerical artefacts, but to realistic capillary waves that have been observed also experimentally (e.g. [16], [19]).

In the second case of Figure 7, with the further increase of the IPD to $\Delta P=45 \mathrm{~Pa}$ (Figure 7(b)), apart from the previously observed capillary waves (that are still evident but without being as intense as in the first case), the interface at the trailing edge of the bubble fluctuates significantly, with the maximum fluctuation located at the longitudinal axis of the cylindrical channel. At time $t=59.6 \mathrm{~ms}$ these fluctuations lead to the formation of small spherical bubbles that start disintegrating successively from the fluctuating interface. A zoomed section has been added to the left of each time instance, since the detached bubble in this case is significantly small. The time instance of $t=59.6 \mathrm{~ms}$ marks the first of a series of successive small bubbles detaching from the same point of the leading elongated TB. This type of partial bubble break-up constitutes the second of the three in total observed break-up regimes. This phenomenon is referred as "Partial Break-up (PB)" regime for the rest of the present paper and corresponds to observation B in the experimental snapshots of Figure 1. It should be highlighted here that as it can be seen from the mesh independency study in Section 3.2. the mesh is fine enough to accurately capture such small interfacial features. This fact, in combination with the experimental observations of [16] and [19], confirms that such small satellite bubbles constitute physical events and not numerical artefacts.

Finally, the further increase of the IPD to $\triangle P=70 \mathrm{~Pa}$ (Figure 7(c)), leads to the formation of a liquid jet that starts to progressively penetrate the bubble from its trailing edge. This results at the end, to the encapsulation of a liquid droplet within the TB. The further, continuous wobbling of the proposed encapsulated liquid droplet in the radial direction, at $t=60.5 \mathrm{~ms}$, breaks the liquid film that separates the TB from the channel wall, leading to a complete break-up of the TB into an elongated leading part and a shorter trailing part. This break-up regime constitutes the third of the overall observed regimes, and it is referred to as "Full Break-up (FB)" regime and it corresponds to observation A in the experimental snapshots of Figure 1. In the experiments of [16], [19] (Figure 1, observation A) the high speed images do not reveal what is happening inside the bubble and only the end result of the full-break up of the bubble can be seen. However, such particular mechanisms where drops travel inside the bubble have been observed in the past both numerically and experimentally and they are reported in [32].

Among all of the 89 in total simulated cases, only these three regimes are identified. From the overall analysis of the IPD variation runs, for the two different channels and the two different fluids simulated, it is characteristic that increasing the IPD value progressively leads to a regime change from NB to PB and FB. This implies that the IPD and therefore the flow acceleration has a direct and significant effect in the resulting interface dynamics of the considered TBs. Another interesting conclusion from the overall observation and analysis of the results is that the increase of the IPD value leads to an increase in the length of the accelerating TBs. This is due to the fact that the 
increase of the IPD leads to a consequent increase in the acceleration of the bubble within the channel that results in an elongation, due to the opposing drag force. All of the considered dimensionless numbers are affected directly or indirectly by the variation of the IPD. Therefore, a mapping of the resulting flow regimes is conducted with respect to the resulting key dimensionless parameters in Section 4.5, at the end of the present paper.



(a)



(b)



(c)

Figure 7. The effect of the IPD on the resulting elongated vapour slug break-up regime (FC-72, $D_{\text {channel }}=3 \mathrm{~mm}, \Delta_{\text {film }}=50 \mu \mathrm{m}, \sigma$ $=0.0084 \mathrm{~N} / \mathrm{m}, q^{\prime \prime}=0.0 \mathrm{~W} / \mathrm{m}^{2}$ ). Dimensionless number values: (a) $F r^{*}=17.22, W e=91.85, C a=0.0196$ and $R e=4698$, (b) $F r^{*}=13.38, W e=214.11, C a=0.0299$ and $R e=7173$, (c) $F r^{*}=12.81, W e=318.92, C a=0.0364$ and $R e=8754$.

\subsection{Effect of Surface Tension (ST)}

Since the surface tension is directly linked to the interface resistance to break-up events, it was also selected as one of the fundamental parameters for the present parametric numerical investigation. In Figure 8, the effect of surface tension is illustrated, using indicatively three cases with a different ST, for the $3 \mathrm{~mm}$ diameter mini-channel. As previously, the proposed figure shows successive time instances of the accelerating TB within the mini-channel, in each case. In more detail, Figures 8 (a), (b) and (c) depict the spatial and temporal evolution of the considered TB when the ST is $0.0084 \mathrm{~N} / \mathrm{m}^{2}, 0.0168 \mathrm{~N} / \mathrm{m}^{2}$ and $0.0252 \mathrm{~N} / \mathrm{m}^{2}$, respectively. The resulting dimensionless parameters for each case are indicated in the figure caption. Four successive time instances of the accelerating TB within the mini-channel are shown in each case. As in the previous sub-section (Figure 7), velocity magnitude contours are also superimposed in a central, longitudinal section within the channel. The black coloured line marks the liquid/vapour interface on the same 
central, longitudinal section. The 3D interface is shown with an opacity of just $35 \%$ in order to visualise the velocity magnitude within the bubble, at the proposed section. The distance from the inlet of the considered channel is also indicated in each case.



(a)



(b)

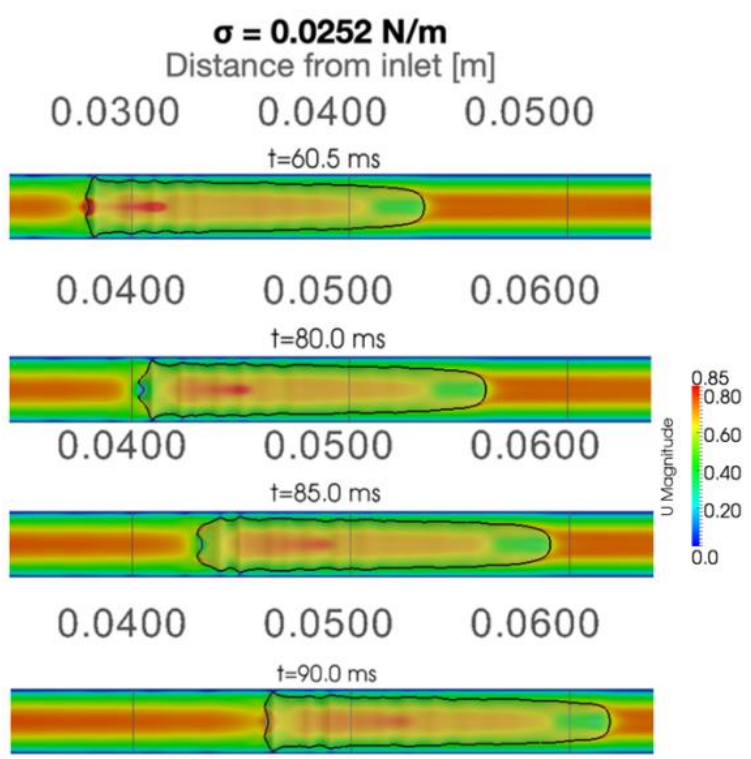

(c)

Figure 8. The effect of the ST on the resulting elongated vapour slug break-up regime (FC-72, $D_{\text {channel }}=3 \mathrm{~mm}, \Delta_{\text {film }}=50 \mu \mathrm{m}$, $\Delta P=70 \mathrm{~Pa}, q^{\prime \prime}=0.0 \mathrm{~W} / \mathrm{m}^{2}$ ). Dimensionless number values: (a) $F r^{*}=12.81, W e=318.92, C a=0.0364$ and $R e=8754$, (b) $F r^{*}=12.81$, $W e=159.46, C a=0.0182$ and $R e=8754$, (c) $F r^{*}=12.81, W e=106.31, C a=0.0121$ and $R e=8754$.

In all three cases, the working fluid, the ILFT, the IPD and the AHF are kept constant, while the ST of the base case is "virtually" doubled and tripled $\left(D_{\text {channel }}=3 \mathrm{~mm}\right.$, FC-72, $\left.\Delta_{\text {film }}=50 \mu \mathrm{m}, \Delta P=70 \mathrm{~Pa}, q "=0.0 \mathrm{~W} / \mathrm{m}^{2}\right)$. The overall ST values examined in the present parametric numerical investigation for the specific IPD (70 Pa) and channel diameter $(3 \mathrm{~mm})$ range from $0.002 \mathrm{~N} / \mathrm{m}$ up to $0.035 \mathrm{~N} / \mathrm{m}$ with selected values calculated, as the ST of FC-72 at the considered initial pressure of the base case multiplied by a factor that ranged from 0.25 up to 4.2. Similar ST value ranges were used also for the $2 \mathrm{~mm}$ channel and for other values of IPD as well as for Ethanol as the working fluid. The details of all these cases along with the resulting dimensionless parameters are included in Table A of the Appendix. 
As it can be observed from Figure 8, as the ST increases the initially observed FB regime, successively shifts to $\mathrm{PB}$ and NB, respectively. This trend is also observed for other IPD values as well as for the $2 \mathrm{~mm}$ diameter channel cases and also for cases where the working fluid is Ethanol. This justifies the initial assumption that the increase of the surface tension should have a direct and significant effect in the resulting break-up regimes, by progressively increasing the resistance of the bubble to break-up events. At, this point it should be mentioned that the We and $\mathrm{Ca}$ numbers are affected directly by the variation of the ST. However, it seems that the variation of the We number is more important for the transition from one break-up regime to the other than the $C a$ number. Therefore, the most relevant mapping of the resulting flow regimes is conducted with respect to the most important dimensionless numbers in Section 4.5, at the end of the present paper.

Another interesting conclusion from the overall observation and analysis of the results for the ST variation is that the increase of the ST value leads to a decrease in the length of the accelerating TBs. Finally, it is characteristic that in the $\mathrm{PB}$ case a vapour pulse in the form of a jet is formed within the bubble just after the PB event that progressively travels to the leading edge of the bubble (Figure 8 (b)). This pulse is also evident in Figure 7(b), but in a much smaller scale as the detached bubble in that particular case is much smaller as the one that is detached in the case of Figure 8(b).

\subsection{Effect of Applied Heat Flux (AHF)}

From the previous sub-sections, it is evident that the identified break-up regimes are governed by the hydrodynamic characteristics of the considered two-phase flows. In more detail, from the overall considered dimensionless parameters the variation in We number as well as in a modified $\mathrm{Fr}$ number $\left(\mathrm{Fr}^{*}\right)$ seems to greatly affect the transition from one break-up regime to the other. $\mathrm{Re}$ and $\mathrm{Ca}$ do not seem to be the controlling dimensionless numbers for the resulting break-up regimes. However, since the motivation of the present investigation is the break-up regimes observed in PHPs (where also diabatic TB flows are encountered), it was deemed appropriate to investigate if these hydro-dynamically generated identified break-up regimes also hold, in the case of parallel liquid film evaporation due to the application of a heat flux at the wall of the considered channels. For this purpose, six of the previously considered adiabatic cases were repeated with two different values of heat flux $\left(q^{\prime \prime}=5000 \mathrm{~W} / \mathrm{m}^{2}\right.$ and $\left.q^{\prime \prime}=10000 \mathrm{~W} / \mathrm{m}^{2}\right)$ applied at the heated part of the considered mini-channel. In Figure 9, the proposed effect is depicted for one of these 6 adiabatic cases, indicatively. In each of the depicted simulations in Figure 9, the working fluid, the IPD, the ST and the ILFT are kept constant (FC-72, $\Delta_{\text {film }}=50 \mu \mathrm{m}, D_{\text {channel }}=3 \mathrm{~mm}, \Delta P=60 \mathrm{~Pa}, \sigma=0.0084 \mathrm{~N} / \mathrm{m}$ ). Therefore, the considered hydrodynamic dimensionless parameters are not altered. Their values are indicated in the figure caption.



Figure 9. The effect of the AHF in the heated section of the considered mini-channel on the resulting elongated vapour slug break-up regime $\left(\mathrm{FC}-72, \Delta_{\text {film }}=50 \mu \mathrm{m}, D_{\text {channel }}=3 \mathrm{~mm}, \Delta P=60 \mathrm{~Pa}, \sigma=0.0084 \mathrm{~N} / \mathrm{m}\right)$. The vapour volume within the channel is also noted for each case. Dimensionless number values: $F r^{*}=13.3, W e=283.62, C a=0.0344$ and $R e=8255$. 
As it can be observed, it is quite interesting that, despite the significant increase in the AHF and the corresponding considerable increase in the resulting volume of the vapour, due to the progressively higher liquid film evaporation, by $7.3 \%$ and $12.9 \%$ for AHFs of $q^{\prime \prime}=5000 \mathrm{~W} / \mathrm{m}^{2}$ and $q^{\prime \prime}=10000 \mathrm{~W} / \mathrm{m}^{2}$, respectively, the bubbles in all three cases experience the same break-up regime (FB) and at similar time instances. This constitutes an indication that the three identified break-up regimes in the overall parametric analysis and for the considered AHFs are indeed related only to the generated hydro-dynamic instabilities of the liquid film and the other observed inertial phenomena that are the result of the counteraction of the global two-phase flow acceleration and the surface tension force. It should be mentioned that this observation was consistent for all of the six selected cases. However, in order to generalise this conclusion, higher heat fluxes need to be investigated in the future, to check if a much more significant increase in the volume of the TB (i.e. >12.9\%) due to higher liquid film evaporation rates, can alter the resulting break-up regime.

\subsection{Effect of Initial Liquid Film Thickness (ILFT)}

Finally, since for the simulations that are conducted for the purposes of the present investigation the ILFT needs to be applied as an assumed initial condition, it was considered appropriate that it should also constitute part of the present parametric investigation. This was done in order to check mainly the sensitivity of the numerical simulation results against this assumption and make sure that the finally selected ILFT value for the overall parametric numerical investigation does not influence the numerical predictions. For this purpose, four different values were tested, $\left(\Delta_{f i l m}=12,25,50,75,270 \mu \mathrm{m}\right)$, for the $3 \mathrm{~mm}$ diameter channel and for an IPD value of $\Delta P=$ 90Pa, using FC-72 as the working fluid. In Figure 10, the effect of ILFT for these five cases is illustrated. It should be mentioned that from the experimental observations of [16], [19] it is estimated that the liquid film thickness varies approximately from $25 \mu \mathrm{m}$ up to $65 \mu \mathrm{m}$. Figure 10 shows a specific time instance of the accelerating TB within the mini-channel, in each case. Velocity magnitude contours are also superimposed in a central, longitudinal section within the channel. The black coloured line marks the liquid/vapour interface on the same central longitudinal section. The 3D interface is shown with an opacity of just $35 \%$ in order to visualise the velocity magnitude within the bubble, at the proposed section.

Figure 10. The effect of the ILFT on the resulting elongated vapour slug break-up regime (FC-72, $\Delta_{\text {film }}=50 \mu \mathrm{m}, D_{\text {channel }}=3 \mathrm{~mm}$, $\Delta P=90 \mathrm{~Pa}, \sigma=0.0084 \mathrm{~N} / \mathrm{m}, q^{\prime \prime}=0.0 \mathrm{~W} / \mathrm{m}^{2}$ ). Dimensionless number values: $F r^{*}=12.85, W e=411.26, C a=0.0414$ and $R e=9941$.

For relatively small values of the ILFT $\left(\Delta_{f i l m}=12-50 \mu \mathrm{m}\right)$, the predicted break-up characteristic does not show any variation, since at exactly the same time instance, after the initiation of the two-phase flow ( $t=62 \mathrm{~ms}$ ), an almost identical liquid jet penetration that leads to a FB regime is observed. However, for further increase of the ILFT value, the resulting effect starts to become progressively more significant $\left(\Delta_{\text {film }}=75 \mu \mathrm{m}\right.$ and $\left.\Delta_{\text {film }}=270 \mu \mathrm{m}\right)$. Therefore, a liquid film of $50 \mu \mathrm{m}$ has been adopted for the simulations of the main parametric analysis (i.e. effects of IPD, ST and AHF). After the effect of the ILFT starts appearing (i.e. $\Delta_{\text {film }}>50 \mu \mathrm{m}$ ), the increase of the ILFT value leads to a successive transition from a FB regime towards a NB regime and to a progressively shorter TB. This might be attributed to the fact that as the ILFT increases, the TB initial volume progressively becomes smaller, up to a limiting value where the effect of surface tension changes, altering the resulting regime (i.e. $\Delta_{\text {film }}=75 \mu \mathrm{m}$ ).

\subsection{Dimensional Analysis}

In order to quantify the global flow conditions that generate the previously identified main break-up regimes, as mentioned previously, a dimensional analysis has also been conducted. As it can be observed from Figure 11, the identified break-up regimes are grouped together forming a clear flow map using the Weber $(W e)$ number and the modified Froude number $\left(F r^{*}\right)$. As it can be seen in Figure 11, a central region where the bubbles undergo a PB regime is identified with a bottom region of NB regime and a top region of FB regime. The red and green circles indicate the selected six adiabatic cases that were reproduced with an applied heat flux of 5,000 and $10,000 \mathrm{~W} / \mathrm{m}^{2}$, respectively. As it can be seen, all these heated cases end up with exactly the same regime as the corresponding adiabatic runs. 




Figure 11. Flow map with different TB break-up regimes for $D_{\text {channel }}=3 \mathrm{~mm}$ and $D_{\text {channel }}=2 \mathrm{~mm}$. Weber number versus a modified Froude number.

In contrast with previous investigations [17], where the focus is usually on the contraction of flow maps where the prevailing two-phase flow regimes are classified (including the TB flow regime), here, for the first time, the focus is to identify and classify break-up regimes that occur in accelerating TB flows in a single accelerating TB. As it can be observed, for the examined flow characteristics and conditions, clear critical values for $W e$ and $\mathrm{Fr}^{*}$ are revealed that predict the boundaries among the thee identified break-up regimes. It is important to notice that the resulted map contains data from two different channel diameters as well as from different working fluids.

\section{Conclusions}

In the present work, an enhanced VOF-based numerical simulation framework is applied for the conduction of parametric numerical experiments, aiming to investigate the effect of various fundamental controlling parameters in the generated break-up phenomena for the case of accelerated Taylor Bubbles within circular minichannels. Two different channel diameters and working fluids are used as the base cases for the parametric numerical investigation. The rest of the runs constitute variation of the imposed pressure difference, the surface tension, the applied heat flux and the initial liquid film thickness.

Three break-up regimes are defined: a Full Break-up regime, a Partial Break-up regime and a No Break-up regime. A new phenomenon, which is very difficult to be observed by the experiments, is revealed, such as the entrainment of liquid droplets at the trailing edge of the TBs. This leads to a bubble break-up (Full Break-up 
regime). Such particular mechanisms, where drops travel inside TBs, have been observed and reported also in the past both numerically and experimentally. However, more tailored experiments are needed in the future to quantify such uncommon mechanisms.

Due to the very small spatial and temporal scales, what is possible to notice with the numerical simulations, which is difficult to be measured and quantified by experiments, is the presence of important capillary waves of the liquid film surrounding the Taylor Bubbles. Another very interesting finding is the fact that the chosen values of the applied heat flux do not influence the resulting break-up regimes and their main spatial and temporal characteristics.

Finally, a dimensional analysis leads to the grouping of the identified break-up regimes according to the Weber number and a modified "pressure" Froude number. Even if in present modern experimental campaigns, it is still difficult to measure fast pressure variations in small lengths of mini-channels, in the future this could be a way to characterise the occurrence of one break-up regime with respect to another one. On the other side, the value of $W e_{c r, N B}=75$ under which no bubble break-up occurs, and the value of $W e_{c r, F B}=250$ above which full bubble break-up occurs are already very important information for conditional sub-models that can be incorporated in 1-D simulation tools in order to enhance their performance by implicitly accounting for prevailing Taylor Bubble break-up phenomena.

\section{Acknowledgements}

The Authors would like to acknowledge UK's Engineering and Physical Science Research Council support through the grant EP/P013112/1 as well as the ESA MAP Projects TOPDESS and ENCOM4. The Authors would also like to acknowledge the contribution of the High-Performance Computing facility of the School of Computing Engineering and Mathematics, in the University of Brighton.

\section{References}

[1] M. Ghiaasiaan, S, Two-Phase Flow, Boiling, and Condensation: In Conventional and Miniature Systems, Second. Cambridge, UK: Cambridge University Press, 2017.

[2] K. A. Triplett, S. M. Ghiaasiaan, S. I. Abdel-Khalik, and D. L. Sadowski, "Gas-liquid two-phase flow in microchannels Part I: two-phase flow patterns," Int. J. Multiph. Flow, vol. 25, no. 3, pp. 377-394, Apr. 1999.

[3] A. M. Barajas and R. L. Panton, "The effects of contact angle on two-phase flow in capillary tubes," Int. J. Multiph. Flow, vol. 19, no. 2, pp. 337-346, Apr. 1993.

[4] A. Serizawa, Z. Feng, and Z. Kawara, "Two-phase flow in microchannels," Exp. Therm. Fluid Sci., vol. 26, no. 6-7, pp. 703-714, Aug. 2002.

[5] T. Cubaud and C.-M. Ho, "Transport of bubbles in square microchannels," Phys. Fluids, vol. 16, no. 12, pp. 4575-4585, Dec. 2004.

[6] S. Haase, "Characterisation of gas-liquid two-phase flow in minichannels with co-flowing fluid injection inside the channel, part II: gas bubble and liquid slug lengths, film thickness, and void fraction within Taylor flow," Int. J. Multiph. Flow, vol. 88, pp. 251-269, Jan. 2017.

[7] A. Peddu, S. Chakraborty, and P. Kr. Das, "Visualization and flow regime identification of downward airwater flow through a $12 \mathrm{~mm}$ diameter vertical tube using image analysis," Int. J. Multiph. Flow, vol. 100, pp. 1-15, Mar. 2018.

[8] C. Yao, J. Zheng, Y. Zhao, Q. Zhang, and G. Chen, "Characteristics of gas-liquid Taylor flow with different liquid viscosities in a rectangular microchannel," Chem. Eng. J., vol. 373, pp. 437-445, Oct. 2019.

[9] J. M. van Baten and R. Krishna, "CFD simulations of mass transfer from Taylor bubbles rising in circular capillaries," Chem. Eng. Sci., vol. 59, no. 12, pp. 2535-2545, Jun. 2004.

[10] T. Taha and Z. F. Cui, "Hydrodynamics of slug flow inside capillaries," Chem. Eng. Sci., vol. 59, no. 6, pp. 1181-1190, Mar. 2004.

[11] R. Gupta, D. F. Fletcher, and B. S. Haynes, "On the CFD modelling of Taylor flow in microchannels," Chem. Eng. Sci., vol. 64, no. 12, pp. 2941-2950, 2009.

[12] A. N. Asadolahi, R. Gupta, D. F. Fletcher, and B. S. Haynes, "CFD approaches for the simulation of hydrodynamics and heat transfer in Taylor flow," Chem. Eng. Sci., vol. 66, no. 22, pp. 5575-5584, Nov. 2011.

[13] E. Lizarraga-Garcia, J. Buongiorno, E. Al-Safran, and D. Lakehal, "A broadly-applicable unified closure relation for Taylor bubble rise velocity in pipes with stagnant liquid," Int. J. Multiph. Flow, vol. 89, pp. 345358, Mar. 2017.

[14] H. Shaban and S. Tavoularis, "Detached eddy simulations of rising Taylor bubbles," Int. J. Multiph. Flow, vol. 
107, pp. 289-300, Oct. 2018.

[15] H. Qiu and X. Wang, "Experimental Study on Interfacial Film Dynamics of Oscillating Multiphase Micro Flows," in 13th Int Symp on Applications of Laser Techniques to Fluid Mechanics, 2006.

[16] D. Mangini, M. Mameli, A. Georgoulas, L. Araneo, S. Filippeschi, and M. Marengo, "A pulsating heat pipe for space applications: Ground and microgravity experiments," Int. J. Therm. Sci., vol. 95, 2015.

[17] L. Pietrasanta et al., "Developing flow pattern maps for accelerated two-phase capillary flows," Exp. Therm. Fluid Sci., vol. 112, p. 109981, Apr. 2020.

[18] V. S. Nikolayev and M. Marengo, "Chapter 2: Pulsating Heat Pipes: Basics of Functioning and Modeling," Encycl. Two-Phase Heat Transf. Flow IV, pp. 63-139, 2018.

[19] D. Mangini, M. Mameli, D. Fioriti, S. Filippeschi, L. Araneo, and M. Marengo, "Hybrid Pulsating Heat Pipe for space applications with non-uniform heating patterns: Ground and microgravity experiments," Appl. Therm. Eng., no. Id, Jan. 2017.

[20] “OpenFOAM, The Open Source CFD Toolbox, User Guide," no. v. 2.2.1, 2013.

[21] A. Georgoulas, P. Koukouvinis, M. Gavaises, and M. Marengo, "Numerical investigation of quasi-static bubble growth and detachment from submerged orifices in isothermal liquid pools: The effect of varying fluid properties and gravity levels," Int. J. Multiph. Flow, vol. 74, pp. 59-78, 2015.

[22] A. Georgoulas, M. Andredaki, and M. Marengo, "An Enhanced VOF Method Coupled with Heat Transfer and Phase Change to Characterise Bubble Detachment in Saturated Pool Boiling," Energies, vol. 10, no. 3, p. 272, Feb. 2017.

[23] E. Teodori, P. Pontes, A. Moita, A. Georgoulas, M. Marengo, and A. Moreira, "Sensible heat transfer during droplet cooling: Experimental and numerical analysis," Energies, vol. 10, no. 6, 2017.

[24] M. Andredaki, A. Georgoulas, N. Miche, and M. Marengo, "NUMERICAL INVESTIGATION OF LIQUID FILM INSTABILITIES AND EVAPORATION IN CONFINED OSCILLATING SLUG-PLUG FLOWS," WIT Trans. Eng. Sci. Comput. Exp. Methods Multiph. Complex Flow X, vol. 123, pp. 127-138, 2019.

[25] K. Vontas, M. Andredaki, A. Georgoulas, K. S. Nikas, and M. Marengo, "Numerical Investigation of Droplet Impact on Smooth Surfaces with Different Wettability Characteristics: Implementation of a dynamic contact angle treatment in OpenFOAM," in 28th European Conference on Liquid Atomization and Spray Systems, 2017.

[26] J. . Brackbill, D. . Kothe, and C. Zemach, "A continuum method for modeling surface tension," J. Comput. Phys., vol. 100, no. 2, pp. 335-354, Jun. 1992.

[27] D. A. Hoang, V. van Steijn, L. M. Portela, M. T. Kreutzer, and C. R. Kleijn, "Benchmark numerical simulations of segmented two-phase flows in microchannels using the Volume of Fluid method," Comput. Fluids, vol. 86, pp. 28-36, Nov. 2013.

[28] S. S. Deshpande, L. Anumolu, and M. F. Trujillo, "Evaluating the performance of the two-phase flow solver interFoam," Comput. Sci. Discov., vol. 5, no. 1, p. 014016, Nov. 2012.

[29] S. Hardt and F. Wondra, "Evaporation model for interfacial flows based on a continuum-field representation of the source terms," J. Comput. Phys., vol. 227, no. 11, pp. 5871-5895, 2008.

[30] S. Arias, D. Legendre, and R. González-Cinca, "Numerical simulation of bubble generation in a T-junction," Comput. Fluids, vol. 56, pp. 49-60, 2012.

[31] A. Georgoulas, P. Koukouvinis, M. Gavaises, and M. Marengo, "Numerical investigation of quasi-static bubble growth and detachment from submerged orifices in isothermal liquid pools: The effect of varying fluid properties and gravity levels," Int. J. Multiph. Flow, vol. 74, 2015.

[32] T. Adabie, "Hydrodynamics of gas-liquid Taylor flow in microchannels," INP Toulouse, 2013. 


\section{Appendix}

Table A. Main characteristics of overall conducted numerical simulations.

\begin{tabular}{|c|c|c|c|c|c|c|c|c|c|c|c|}
\hline & $\begin{array}{l}\text { ILFT } \\
{[\mu \mathrm{m}]}\end{array}$ & $\begin{array}{l}\text { IPD } \\
{[\mathrm{Pa}]}\end{array}$ & $\begin{array}{c}\mathrm{ST} \\
{[\mathrm{N} / \mathrm{m}]}\end{array}$ & $\begin{array}{c}\rho \\
{\left[\mathrm{kg} / \mathrm{m}^{3}\right.} \\
]\end{array}$ & $\begin{array}{c}\mu \\
{[\mathrm{kg} /(\mathrm{ms})]}\end{array}$ & $\begin{array}{c}\mathrm{D}_{\text {channel }} \\
{[\mathrm{m}]}\end{array}$ & $\mathrm{Fr} *$ & We & $\mathrm{Ca}$ & $\mathbf{R e}$ & $\begin{array}{l}\text { Break-up } \\
\text { Regime }\end{array}$ \\
\hline 1 & 50 & 15 & 0.0084381 & 1621.1 & 0.000413267 & 0.003 & 17.22 & 91.85 & 0.0196 & 4698 & no break-up \\
\hline 2 & 50 & 30 & 0.0084381 & 1621.1 & 0.000413267 & 0.003 & 14.27 & 152.17 & 0.0252 & 6047 & $\begin{array}{c}\text { partial } \\
\text { break-up }\end{array}$ \\
\hline 3 & 50 & 45 & 0.0084381 & 1621.1 & 0.000413267 & 0.003 & 13.38 & 214.11 & 0.0299 & 7173 & $\begin{array}{c}\text { partial } \\
\text { break-up }\end{array}$ \\
\hline 4 & 50 & 50 & 0.0084381 & 1621.1 & 0.000413267 & 0.003 & 14.15 & 251.5 & 0.0324 & 7774 & break-up \\
\hline 5 & 50 & 60 & 0.0084381 & 1621.1 & 0.000413267 & 0.003 & 13.3 & 283.62 & 0.0344 & 8255 & break-up \\
\hline 6 & 50 & 65 & 0.0084381 & 1621.1 & 0.000413267 & 0.003 & 12.76 & 294.77 & 0.035 & 8416 & break-up \\
\hline 7 & 50 & 70 & 0.0084381 & 1621.1 & 0.000413267 & 0.003 & 12.81 & 318.92 & 0.0364 & 8754 & break-up \\
\hline 8 & 50 & 75 & 0.0084381 & 1621.1 & 0.000413267 & 0.003 & 14.37 & 383.13 & 0.0399 & 9595 & break-up \\
\hline 9 & 50 & 90 & 0.0084381 & 1621.1 & 0.000413267 & 0.003 & 12.85 & 411.26 & 0.0414 & 9941 & break-up \\
\hline 10 & 50 & 90 & 0.0168762 & 1621.1 & 0.000413267 & 0.003 & 12.85 & 205.63 & 0.0207 & 9941 & break-up \\
\hline 11 & 50 & 90 & 0.0253143 & 1621.1 & 0.000413267 & 0.003 & 12.85 & 137.09 & 0.0138 & 9941 & break-up \\
\hline 12 & 50 & 90 & 0.0337524 & 1621.1 & 0.000413267 & 0.003 & 12.85 & 102.81 & 0.0103 & 9941 & no break-up \\
\hline 13 & 50 & 90 & 0.0506286 & 1621.1 & 0.000413267 & 0.003 & 12.85 & 68.54 & 0.0069 & 9941 & no break-up \\
\hline 14 & 270 & 75 & 0.0084381 & 1621.1 & 0.000413267 & 0.003 & 14.37 & 383.13 & 0.0399 & 9595 & no break-up \\
\hline 15 & 270 & 90 & 0.0084381 & 1621.1 & 0.000413267 & 0.003 & 12.85 & 411.26 & 0.0414 & 9941 & no break-up \\
\hline 16 & 270 & 90 & 0.0168762 & 1621.1 & 0.000413267 & 0.003 & 12.85 & 205.63 & 0.0207 & 9941 & $\begin{array}{c}\text { partial } \\
\text { break-up }\end{array}$ \\
\hline 17 & 270 & 90 & 0.0337524 & 1621.1 & 0.000413267 & 0.003 & 12.85 & 102.81 & 0.0103 & 9941 & $\begin{array}{c}\text { partial } \\
\text { break-up }\end{array}$ \\
\hline 18 & 270 & 90 & 0.0506286 & 1621.1 & 0.000413267 & 0.003 & 12.85 & 68.54 & 0.0069 & 9941 & $\begin{array}{c}\text { partial } \\
\text { break-up }\end{array}$ \\
\hline 19 & 12 & 90 & 0.0084381 & 1621.1 & 0.000413267 & 0.003 & 12.85 & 411.26 & 0.0414 & 9941 & break-up \\
\hline 20 & 25 & 90 & 0.0084381 & 1621.1 & 0.000413267 & 0.003 & 12.85 & 411.26 & 0.0414 & 9941 & break-up \\
\hline 21 & 75 & 90 & 0.0084381 & 1621.1 & 0.000413267 & 0.003 & 12.85 & 411.26 & 0.0414 & 9941 & break-up \\
\hline 22 & 50 & 60 & 0.0168762 & 1621.1 & 0.000413267 & 0.003 & 13.3 & 141.81 & 0.0172 & 8255 & $\begin{array}{c}\text { partial } \\
\text { break-up }\end{array}$ \\
\hline 23 & 50 & 60 & 0.0253143 & 1621.1 & 0.000413267 & 0.003 & 13.3 & 94.54 & 0.0115 & 8255 & $\begin{array}{c}\text { partial } \\
\text { break-up }\end{array}$ \\
\hline 24 & 270 & 90 & 0.00675048 & 1621.1 & 0.000413267 & 0.003 & 12.85 & 514.07 & 0.0517 & 9941 & $\begin{array}{c}\text { partial } \\
\text { break-up }\end{array}$ \\
\hline 25 & 270 & 90 & 0.00759429 & 1621.1 & 0.000413267 & 0.003 & 12.85 & 456.95 & 0.046 & 9941 & no break-up \\
\hline 26 & 50 & 65 & 0.0168762 & 1621.1 & 0.000413267 & 0.003 & 12.76 & 147.38 & 0.0175 & 8416 & $\begin{array}{c}\text { partial } \\
\text { break-up }\end{array}$ \\
\hline 27 & 50 & 65 & 0.0253143 & 1621.1 & 0.000413267 & 0.003 & 12.76 & 98.26 & 0.0117 & 8416 & $\begin{array}{c}\text { partial } \\
\text { break-up }\end{array}$ \\
\hline 28 & 50 & 70 & 0.0168762 & 1621.1 & 0.000413267 & 0.003 & 12.81 & 159.46 & 0.0182 & 8754 & break-up \\
\hline 29 & 50 & 70 & 0.0253143 & 1621.1 & 0.000413267 & 0.003 & 12.81 & 106.31 & 0.0121 & 8754 & no break-up \\
\hline 30 & 50 & 50 & 0.0168762 & 1621.1 & 0.000413267 & 0.003 & 14.15 & 125.75 & 0.0162 & 7774 & $\begin{array}{c}\text { partial } \\
\text { break-up }\end{array}$ \\
\hline
\end{tabular}




\begin{tabular}{|c|c|c|c|c|c|c|c|c|c|c|c|}
\hline 31 & 50 & 50 & 0.0253143 & 1621.1 & 0.000413267 & 0.003 & 14.15 & 83.83 & 0.0108 & 7774 & $\begin{array}{c}\text { partial } \\
\text { break-up }\end{array}$ \\
\hline 32 & 50 & 50 & 0.00675048 & 1621.1 & 0.000413267 & 0.003 & 14.15 & 314.37 & 0.0404 & 7774 & break-up \\
\hline 33 & 50 & 65 & 0.00675048 & 1621.1 & 0.000413267 & 0.003 & 12.76 & 368.46 & 0.0438 & 8416 & break-up \\
\hline 34 & 50 & 70 & 0.00675048 & 1621.1 & 0.000413267 & 0.003 & 12.81 & 398.65 & 0.0455 & 8754 & break-up \\
\hline 35 & 50 & 15 & 0.00168762 & 1621.1 & 0.000413267 & 0.003 & 17.22 & 459.24 & 0.0978 & 4698 & no break-up \\
\hline 36 & 50 & 15 & 0.00506286 & 1621.1 & 0.000413267 & 0.003 & 17.22 & 153.08 & 0.0326 & 4698 & no break-up \\
\hline 37 & 50 & 20 & 0.00210952 & 1621.1 & 0.000413267 & 0.003 & 16.11 & 458.22 & 0.0873 & 5246 & break-up \\
\hline 38 & 50 & 20 & 0.0168762 & 1621.1 & 0.000413267 & 0.003 & 16.11 & 57.28 & 0.0109 & 5246 & no break-up \\
\hline 39 & 50 & 25 & 0.00253143 & 1621.1 & 0.000413267 & 0.003 & 15.06 & 446.2 & 0.0787 & 5671 & break-up \\
\hline 40 & 50 & 25 & 0.00675048 & 1621.1 & 0.000413267 & 0.003 & 15.06 & 167.33 & 0.0295 & 5671 & $\begin{array}{c}\text { partial } \\
\text { break-up }\end{array}$ \\
\hline 41 & 50 & 25 & 0.0168762 & 1621.1 & 0.000413267 & 0.003 & 15.06 & 66.93 & 0.0118 & 5671 & no break-up \\
\hline 42 & 50 & 20 & 0.0025314 & 1621.1 & 0.000413267 & 0.003 & 16.11 & 381.86 & 0.0728 & 5246 & break-up \\
\hline 43 & 50 & 20 & 0.0033752 & 1621.1 & 0.000413267 & 0.003 & 16.11 & 286.39 & 0.0546 & 5246 & break-up \\
\hline 44 & 50 & 20 & 0.0042191 & 1621.1 & 0.000413267 & 0.003 & 16.11 & 229.11 & 0.0437 & 5246 & break-up \\
\hline 45 & 50 & 25 & 0.0042191 & 1621.1 & 0.000413267 & 0.003 & 15.06 & 267.72 & 0.0472 & 5671 & break-up \\
\hline 46 & 50 & 20 & 0.0059067 & 1621.1 & 0.000413267 & 0.003 & 16.11 & 163.65 & 0.0312 & 5246 & $\begin{array}{c}\text { partial } \\
\text { break-up }\end{array}$ \\
\hline 47 & 50 & 20 & 0.0075943 & 1621.1 & 0.000413267 & 0.003 & 16.11 & 127.28 & 0.0243 & 5246 & $\begin{array}{c}\text { partial } \\
\text { break-up }\end{array}$ \\
\hline 48 & 50 & 15 & 0.0084381 & 1621.1 & 0.000413267 & 0.002 & 7.55 & 26.84 & 0.0129 & 2074 & no break-up \\
\hline 49 & 50 & 30 & 0.0084381 & 1621.1 & 0.000413267 & 0.002 & 5.96 & 42.35 & 0.0163 & 2605 & no break-up \\
\hline 50 & 50 & 60 & 0.0084381 & 1621.1 & 0.000413267 & 0.002 & 5.92 & 84.23 & 0.0229 & 3673 & no break-up \\
\hline 51 & 50 & 90 & 0.0084381 & 1621.1 & 0.000413267 & 0.002 & 6.59 & 140.65 & 0.0296 & 4747 & no break-up \\
\hline 52 & 50 & 15 & 0.0084381 & 1621.1 & 0.000413267 & 0.002 & 7.55 & 26.84 & 0.0129 & 2074 & no break-up \\
\hline 53 & 50 & 30 & 0.0084381 & 1621.1 & 0.000413267 & 0.002 & 5.96 & 42.35 & 0.0163 & 2605 & no break-up \\
\hline 54 & 50 & 60 & 0.0084381 & 1621.1 & 0.000413267 & 0.002 & 5.92 & 84.23 & 0.0229 & 3673 & no break-up \\
\hline 55 & 50 & 90 & 0.0084381 & 1621.1 & 0.000413267 & 0.002 & 6.59 & 140.65 & 0.0296 & 4747 & no break-up \\
\hline 56 & 50 & 120 & 0.0084381 & 1621.1 & 0.000413267 & 0.002 & 7.44 & 211.66 & 0.0364 & 5823 & $\begin{array}{c}\text { partial } \\
\text { break-up }\end{array}$ \\
\hline 57 & 50 & 150 & 0.0084381 & 1621.1 & 0.000413267 & 0.002 & 8.36 & 297.28 & 0.0431 & 6901 & break-up \\
\hline 58 & 50 & 180 & 0.0084381 & 1621.1 & 0.000413267 & 0.002 & 9.31 & 397.41 & 0.0498 & 7979 & break-up \\
\hline 59 & 50 & 60 & 0 & 7 & 0. & 0.002 & 1 & 6.76 & 97 & 694 & no break-up \\
\hline 60 & 50 & 90 & 0.017798 & 757.17 & 0.000615466 & 0.002 & 1.43 & 14.47 & 0.0143 & 1015 & no break-up \\
\hline 61 & 50 & 180 & 0.017798 & 757.17 & 0.000615466 & 0.002 & 2.75 & 55.55 & 0.0279 & 1988 & no break-up \\
\hline 62 & 50 & 400 & 0.017798 & 757.17 & 0.000615466 & 0.002 & 6 & 269.58 & 0.0616 & 4380 & break-up \\
\hline 63 & 50 & 60 & 0.035596 & 757.17 & 0.000615466 & 0.003 & 4.63 & 23.4 & 0.0105 & 2235 & no break-up \\
\hline 64 & 50 & 90 & 0.017798 & 757.17 & 0.000615466 & 0.003 & 6.06 & 91.86 & 0.0293 & 3131 & no break-up \\
\hline 65 & 50 & 180 & 0.017798 & 757.17 & 0.000615466 & 0.003 & 10.57 & 320.63 & 0.0548 & 5850 & break-up \\
\hline 66 & 50 & 400 & 0.017798 & 757.17 & 0.000615466 & 0.003 & 21.86 & 1473.7 & 0.1175 & 12541 & break-up \\
\hline 67 & 50 & 180 & 0.035596 & 757.17 & 0.000615466 & 0.003 & 10.57 & 160.31 & 0.0274 & 5850 & $\begin{array}{c}\text { partial } \\
\text { break-up }\end{array}$ \\
\hline
\end{tabular}




\begin{tabular}{|c|c|c|c|c|c|c|c|c|c|c|c|}
\hline 68 & 50 & 180 & 0.026697 & 757.17 & 0.000615466 & 0.003 & 10.57 & 213.75 & 0.0365 & 5850 & $\begin{array}{c}\text { partial } \\
\text { break-up }\end{array}$ \\
\hline 69 & 50 & 60 & 0.0026697 & 757.17 & 0.000615466 & 0.003 & 4.63 & 311.95 & 0.1396 & 2235 & break-up \\
\hline 70 & 50 & 60 & 0.0035596 & 757.17 & 0.000615466 & 0.003 & 4.63 & 233.96 & 0.1047 & 2235 & $\begin{array}{c}\text { partial } \\
\text { break-up }\end{array}$ \\
\hline 71 & 50 & 60 & 0.0035596 & 757.17 & 0.000615466 & 0.002 & 1 & 33.81 & 0.0487 & 694 & no break-up \\
\hline 72 & 50 & 90 & 0.0035596 & 757.17 & 0.000615466 & 0.002 & 1.43 & 72.35 & 0.0713 & 1015 & no break-up \\
\hline 73 & 50 & 180 & 0.0035596 & 757.17 & 0.000615466 & 0.002 & 2.75 & 277.74 & 0.1397 & 1988 & break-up \\
\hline 74 & 50 & 180 & 0.0053394 & 757.17 & 0.000615466 & 0.002 & 2.75 & 185.16 & 0.0931 & 1988 & no break-up \\
\hline 75 & 50 & 120 & 0.017798 & 757.17 & 0.000615466 & 0.003 & 7.54 & 152.47 & 0.0378 & 4034 & $\begin{array}{c}\text { partial } \\
\text { break-up }\end{array}$ \\
\hline 76 & 50 & 150 & 0.017798 & 757.17 & 0.000615466 & 0.003 & 9.05 & 228.69 & 0.0463 & 4940 & $\begin{array}{c}\text { partial } \\
\text { break-up }\end{array}$ \\
\hline 77 & 50 & 180 & 0.0044495 & 757.17 & 0.000615466 & 0.002 & 2.75 & 222.2 & 0.1118 & 1988 & $\begin{array}{c}\text { partial } \\
\text { break-up }\end{array}$ \\
\hline 78 & 50 & 60 & 0.0053394 & 757.17 & 0.000615466 & 0.003 & 4.63 & 155.97 & 0.0698 & 2235 & no break-up \\
\hline 79 & 50 & 60 & 0.0044495 & 757.17 & 0.000615466 & 0.003 & 4.63 & 187.17 & 0.0838 & 2235 & $\begin{array}{c}\text { partial } \\
\text { break-up }\end{array}$ \\
\hline 80 & 50 & 180 & 0.053394 & 757.17 & 0.000615466 & 0.003 & 10.57 & 106.88 & 0.0183 & 5850 & $\begin{array}{c}\text { partial } \\
\text { break-up }\end{array}$ \\
\hline 81 & 50 & 150 & 0.035596 & 757.17 & 0.000615466 & 0.003 & 9.05 & 114.35 & 0.0231 & 4940 & $\begin{array}{c}\text { partial } \\
\text { break-up }\end{array}$ \\
\hline 82 & 50 & 150 & 0.008899 & 757.17 & 0.000615466 & 0.003 & 9.05 & 457.38 & 0.0926 & 4940 & break-up \\
\hline 83 & 50 & 120 & 0.035596 & 757.17 & 0.000615466 & 0.003 & 7.54 & 76.24 & 0.0189 & 4034 & $\begin{array}{c}\text { partial } \\
\text { break-up }\end{array}$ \\
\hline 84 & 50 & 150 & 0.053394 & 757.17 & 0.000615466 & 0.003 & 9.05 & 76.23 & 0.0154 & 4940 & $\begin{array}{c}\text { partial } \\
\text { break-up }\end{array}$ \\
\hline 85 & 50 & 180 & 0.071192 & 757.17 & 0.000615466 & 0.003 & 10.57 & 80.16 & 0.0137 & 5850 & $\begin{array}{c}\text { partial } \\
\text { break-up }\end{array}$ \\
\hline 86 & 50 & 150 & 0.071192 & 757.17 & 0.000615466 & 0.003 & 9.05 & 57.17 & 0.0116 & 4940 & no break-up \\
\hline 87 & 50 & 120 & 0.053394 & 757.17 & 0.000615466 & 0.003 & 7.54 & 50.82 & 0.0126 & 4034 & no break-up \\
\hline 88 & 50 & 180 & 0.08899 & 757.17 & 0.000615466 & 0.003 & 10.57 & 64.13 & 0.011 & 5850 & no break-up \\
\hline 89 & 50 & 180 & 0.106788 & 757.17 & 0.000615466 & 0.003 & 10.57 & 53.44 & 0.0091 & 5850 & no break-up \\
\hline
\end{tabular}

Fr* derivation from Fr number:

$$
\begin{aligned}
& F r=\frac{u}{\sqrt{g L}}=\frac{u}{\sqrt{\frac{F}{m} L}}=\frac{u}{\sqrt{\frac{A \Delta P}{m} L}}=\frac{u}{\sqrt{\frac{A \Delta P}{\rho V} L}}=\frac{u}{\sqrt{\frac{A \Delta P}{\rho(A L)} L}}=\frac{u}{\sqrt{\frac{\Delta P}{\rho}}} \\
& F r^{*}=F r^{2}=\frac{\rho u^{2}}{\Delta P} \frac{\left(\mathrm{kg} / \mathrm{m}^{3}\right) \mathrm{m}^{2} \mathrm{~s}^{-2}}{\mathrm{kgm}^{-1} \mathrm{~s}^{-2}}
\end{aligned}
$$

Where $u$ is the flow velocity and $g$ is the external field. Since the gravitational acceleration is zero in the cases considered in the present paper, $g$ in these cases corresponds to the flow acceleration due to the imposed pressure difference $\Delta P$ between the inlet and the outlet of the channel. $L$ is the length of the channel, $A$ its cross-sectional area and $V$ its volume. 